\title{
Quantity and bioavailability of sediment organic matter as signatures of benthic trophic status
}

\author{
Antonio Pusceddu ${ }^{1, *}$, Antonio Dell'Anno ${ }^{1}$, Mauro Fabiano ${ }^{2}$, Roberto Danovaro ${ }^{1}$ \\ ${ }^{1}$ Department of Marine Sciences, Polytechnic University of Marche, Via Brecce Bianche, 60131 Ancona, Italy \\ ${ }^{2}$ Department for the Study of the Territory and its Resources, University of Genoa, Corso Europa, 26, 16100 Genova, Italy
}

\begin{abstract}
Tools used for assessing marine trophic status are generally based on water column characteristics, which, however, may provide unreliable classification of the benthic trophic status. Here, we provide evidence from the literature that quantity and bioavailability of sediment organic matter are reliable proxies to assess benthic marine trophic status. We compiled data on the protein, carbohydrate and lipid concentration of sediments from different oceanic and coastal regions and varying water depths. The concentration of these 3 components as a whole (biopolymeric carbon) was found to be significantly correlated $(r=0.84)$ with the total organic carbon concentration, suggesting that the biopolymeric fraction is representative of the total organic carbon pool. However, the systematic variation of the biopolymeric fraction was higher than that of total organic carbon concentrations, suggesting that biopolymeric carbon is a more sensitive proxy of benthic trophic status than is the total carbon pool. Furthermore, biopolymeric carbon was significantly correlated to the amount of phytopigments, indicating that biopolymeric carbon accumulation in the sediment is related to inputs of algal carbon. Biopolymeric carbon concentrations were also positively correlated to the sediment community oxygen consumption, suggesting that the progressive accumulation of biopolymeric carbon could be an additional co-factor potentially responsible for hypoxic or anoxic events. The enzymatically digestible and algal fractions of biopolymeric carbon decreased in sediments with increasing biopolymeric carbon content (i.e. eutrophic systems), suggesting that organic carbon in eutrophic sediments is mostly refractory in nature. We propose that a biopolymeric carbon concentration in the sediment of $>2.5 \mathrm{mg} \mathrm{C} \mathrm{g}^{-1}$, being associated with a bioavailable fraction of $<10 \%$, can be considered as a threshold level at which benthic consumers may experience mostly refractory organic carbon.
\end{abstract}

KEY WORDS: Marine sediments · Organic matter · Trophic status

\section{INTRODUCTION}

Eutrophication is a frequent and widespread phenomenon associated with the human utilisation of coastal areas of oceans, and its development is generally described using 'input-response' models (Cloern 2001). These models assume that an increase in nutrient concentrations causes an increase of primary production that, if surpassing certain threshold levels, primes detrimental effects on the ecosystem (Pinckney et al. 2001).

The trophic status of marine ecosystems is generally assessed through chemical measurements (e.g. inorganic nitrogen and phosphorus) and/or surrogate mea- surements of algal biomass in the water column (Stefanou et al. 2000). These proxies may fail in detecting the consequences of increased nutrient loads on benthic systems (Dell'Anno et al. 2002) since they cannot provide any predictive information on the rate of organic matter inputs to the benthos (i.e. benthic trophic status; Jørgensen \& Richardson 1996).

Benthic trophic status can be assessed in terms of total organic carbon (TOC) supply rate to the sea bottom (as $\mathrm{g} \mathrm{C} \mathrm{m}^{-2} \mathrm{yr}^{-1}$; Nixon 1995). However, this is difficult to assess since the magnitude of the organic carbon fluxes is not always directly linked to the primary production in the water column, and the assessment of all of the possible inputs of organic carbon is difficult to 
achieve synoptically on large spatial and temporal scales (Lee et al. 1988). Another approach uses biological indicators such as presence and/or abundance of benthic diatoms and macroalgae (Duarte 1995, Kelly 1998), but the applicability of this method is limited to those systems characterised by the presence or dominance of living photosynthetic primary producers. Moreover, it does not provide any indication of the possible consequences of eutrophication at higher trophic levels of the benthic food web. However, the consequences of changing trophic status reach all hierarchical levels of the ecosystem organisation.

The response of consumers to increased organic matter supply is influenced more by organic matter quality (e.g. bioavailability) rather than by bulk concentration in the ecosystem (Cebrián et al. 1998, Huxel 1999). Thus, the assessment of the trophic status needs to be extended to a more comprehensive description of the organic matter available for heterotrophic nutrition, and should include indicators of the quantity and bioavailability of all (including detrital) resources (Grall \& Chauvaud 2002). This is particularly important for the assessment of the benthic trophic status, where the largest reservoir of food for benthic consumers is deposited (detrital) organic matter (Jørgensen \& Richardson 1996).

Studies aiming at providing a reconstruction of longterm changes in the productivity of a given system have used stratigraphic analyses of organic carbon, nitrogen and phosphorus concentrations coupled with ${ }^{210} \mathrm{~Pb}$ geochronology (Cornwell et al. 1996), foraminifera distributions (Duijnstee et al. 2004), and stable isotopic and lipid biomarker signatures in sediments (Zimmerman \& Canuel 2002). These features allowed an assessment of historical changes in the trophic status of aquatic ecosystems only if analysed as a suite of indicators, since no indicator alone provided adequate information concerning the multiple, interrelated components of the ecosystems (Turner et al. 2006).

Alternatively, recent changes in the benthic trophic status through time can be detected by means of an analysis of organic carbon in superficial sediments. Several studies have provided evidence that eutrophication is associated with a net accumulation or burial of organic carbon in the sediment (Cornwell et al. 1996, Emeis et al. 2000, Farías 2003). Other studies reported decreasing values of the $\mathrm{C} / \mathrm{N}$ ratio in eutrophic sediments (Sampou \& Oviatt 1991).

Organic matter in marine sediments is composed of compounds exhibiting different levels of bioavailability for consumers, ranging from labile (i.e. immediately digestible, sensu Mayer et al. 1995) to refractory (recalcitrant to decomposition). Refractory compounds (such as humic and fulvic acids, structural carbohydrates and 'black' carbon) generally account for most of the sedi- mentary organic matter, and due to very low degradation rates, accumulate in marine sediments (Middelburg et al. 1999). In contrast, highly labile compounds, representing the fraction of organic matter that is rapidly digested by benthic consumers, are subjected to greater temporal and spatial changes (Mayer et al. 1995). However, between these 2 opposite levels of bioavailability, there is a continuum of characteristics, which passes through intermediate levels of bioavailability. Unfortunately, the assessment of the lability of sediment organic carbon is not an easy task, and generally implies several operational assumptions.

The biopolymeric fraction of sediment organic carbon, measured as the sum of protein, carbohydrate and lipid carbon (BPC), has often been reported as the fraction of TOC potentially available to benthic consumers (Fabiano et al. 1995, Bianchelli et al. 2008). More recent investigations reported that only a fraction (5 to $30 \%$ ) of these biopolymers is enzymatically digestible by consumers (Pusceddu et al. 2003).

In this work, we tested the following hypotheses: (1) BPC (sensu Fabiano et al. 1995) is related to TOC in marine sediments, (2) the algal fraction of BPC changes in sediments exhibiting different BPC concentrations, (3) temporal variability in BPC concentration in the sediment changes under different benthic trophic status conditions, (4) sediment community oxygen consumption is related to BPC concentrations and (5) the enzymatically digestible fraction of BPC varies in sediments characterised by different trophic status.

Our goals were, thus, to (1) provide evidence that the quantity and bioavailability of BPC are reliable proxies for marine benthic trophic status, and (2) identify potential threshold levels of key organic carbon-related variables indicating trophic status shifts. To achieve these goals, we examined data from the published literature for relationships between BPC and TOC concentrations in marine sediments from different oceanic and coastal regions and water depths, including intertidal shallow waters and hadal depths. Then we explored the relationships between the quantity of BPC and (1) the algal carbon fraction associated with phytopigments, (2) the sediment community oxygen consumption (SCOC) and (3) the bioavailable (i.e. enzymatically digestible) fraction of sediment organic carbon (BAC).

\section{MATERIALS AND METHODS}

We compiled data published over the last $20 \mathrm{yr}$ on phytopigment (chlorophyll a $[\mathrm{chl}$ a] and phaeopigment), protein, lipid, carbohydrate, BPC and TOC concentrations in surface marine sediments worldwide (Table 1). Because of the multiple different sources of these data, we noticed that not all variables have been 
measured in all studies. All of the data used in this study were obtained from the scientific literature (see Table 1) using similar analytical methods. Possible biases due to different devices used for sediment sampling and/or the use of different analytical protocols were accurately checked (details are described later).

Sediment samples (top $2 \mathrm{~cm}$ ) were collected from coastal to hadal depths (i.e. from 0 to $7800 \mathrm{~m}$ water depth) using manual corers, Van Veen grabs, boxcorers or multiple corers (Table 1). Previous studies have demonstrated that the quantity and biochemical compositions of sediment organic matter collected using multiple corers and box-corers display some differences (Shirayama \& Fukushima 1995, Danovaro et al. 1998). However, since the differences in the concentration of organic matter in sediments collected using different sampling devices are of the same order of magnitude as are the differences in organic matter concentrations determined on sediment replicates collected using the same sampling device (Danovaro et al. 1998), the data used in this study were not corrected.

The concentrations of sedimentary chl aand phaeopigments were determined spectrophotometrically or spectrofluorometrically, according to standard protocols (Lorenzen \& Jeffrey 1980). Total sedimentary lipids were extracted in chloroform:methanol (1:1, vol:vol; Bligh \& Dyer 1959) and the resulting fraction, after evaporation in a dry hot bath at 80 to $100^{\circ} \mathrm{C}$ for $20 \mathrm{~min}$, was quantified according to the sulfuric acid carbonisation procedure (Marsh \& Weinstein 1966). Data on total lipids presented in this study were expressed in tripalmitine equivalents. The lipid fraction determined using this protocol allows the quantification of total lipid concentrations, but does not permit discriminating between different classes of lipids, which is different from chromatographic methods generally used in organic geochemistry (Volkman 2006). However, this was not the main interest of the present study.

Total protein concentrations were determined according to Lowry et al. (1951) as modified by Hartree (1972) and Rice (1982) to compensate for phenol interference and are expressed as bovine serum albumin (BSA) equivalents. Bradford's (1976) protocol, used in some of the studies, provides results similar to Lowry et al.'s (1951) protocol (Berges et al. 1993) and, therefore, no corrections were applied. Total carbohydrate concentrations, expressed in glucose equivalents, were obtained according to the Gerchacov \& Hatcher (1972) protocol based on the phenol and concentrated sulfuric acid reaction with saccharides. The fractions of protein and carbohydrate enzymatically digestible were assessed according to Danovaro et al. (2001), modified for coastal sediments by Pusceddu et al. (2003), and reported as BSA and glucose equivalents, respectively. Briefly, enzymatically digestible proteins were deter- mined as the difference between protein contents of intact sediments and sediments added with a solution of proteinase- $\mathrm{K}$ and protease after a $1 \mathrm{~h}$ incubation at $37^{\circ} \mathrm{C}$. Protein analyses from these samples were carried out according to Lowry et al. (1951). Enzymatically digestible carbohydrates were determined as the difference between carbohydrate quantities released by sediment added with a mixture of enzymes ( $\alpha$-amylase, $\beta$-glucosidase, proteinase-K, lipase) and by intact sediments after $1 \mathrm{~h}$ incubation at room temperature. Carbohydrates from all supernatants and from intact sediments were analyzed according to Gerchacov \& Hatcher (1972). Sediment subsamples muffled at $550^{\circ} \mathrm{C}$ for $4 \mathrm{~h}$ and processed as described previously were used as blanks. Concentrations of enzymatically digestible proteins and carbohydrates were normalized to sediment dry weight.

Total organic $\mathrm{C}$ concentrations in the sediment were determined according to Hedges \& Stern (1984). BPC concentrations were calculated as the sum of protein, carbohydrate and lipid carbon equivalents, using conversion factors obtained from the elemental analysis of standard molecules $\left(0.49,0.4\right.$ and $0.75 \mu \mathrm{g} \mathrm{C} \mathrm{\mu g}^{-1}$, respectively, for BSA, glucose and tripalmitine; Fabiano et al. 1995). Bioavailable carbon (BAC) concentration was calculated as the sum of digestible proteins and carbohydrates converted into carbon equivalents by using the same factors as for their total pools (Danovaro et al. 2001). The algal carbon contribution to BPC was calculated as the percentage of chl a to BPC concentrations, after converting chl a concentration into carbon equivalents using a mean value of $40 \mu \mathrm{g} \mathrm{C}$ $\mu^{-1}$ chl a (Pusceddu et al. 1999).

Statistical analyses. The relationships between the different fractions of sediment organic matter were assessed using either Type-I or Type-II regression analyses (Legendre \& Legendre 1998). In particular, the Type-II regression analysis was carried out to avoid the underestimation of the slope of linear relationships between variables containing errors (Legendre \& Legendre 1998). The Type-II regression analysis was carried out using the major axis regression method, since in all of the cases in the present study the variables were expressed in the same physical units or were dimensionless (Jolicoeur 1990). Type-II regression analyses were performed using the MODEL-II. exe routine (Legendre 2001).

The statistical differences between slopes and intercepts obtained from Type-I regressions testing for the relationships between variables in different environmental contexts were assessed by means of an analysis of covariance (ANCOVA). When slopes were found to be heterogeneous we used Tukey's multiple comparison tests (Zar 1984) to determine which combinations of ecosystems differed. 


\begin{tabular}{|c|c|c|c|c|c|c|c|c|c|c|c|c|c|}
\hline 蒿 & 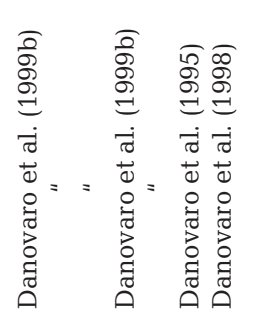 & $=$ & $=$ & 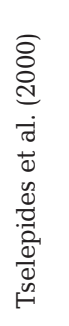 & 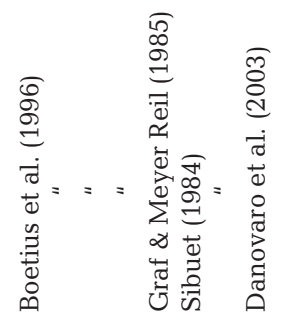 & 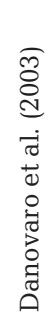 & 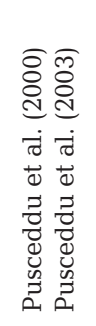 & 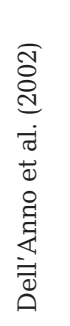 & 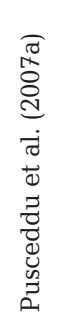 & $=$ & $=$ & $=$ & 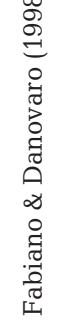 \\
\hline 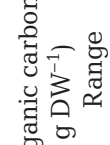 & 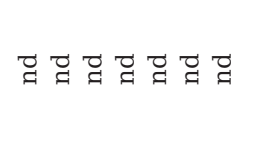 & $\bar{q}$ & $\bar{g}$ & $\begin{array}{l}0 \\
\dot{j} \\
1 \\
0 \\
\text { in }\end{array}$ & 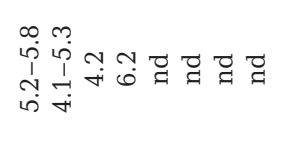 & $\bar{q}$ & $\bar{g} \bar{g}$ & 풀 & $\begin{array}{l}m \\
\infty \\
1 \\
\infty \\
\infty\end{array}$ & $\begin{array}{l}0 \\
\dot{0} \\
\stackrel{1}{1} \\
\stackrel{\sim}{\rightarrow}\end{array}$ & $\begin{array}{l}m \\
\infty \\
0 \\
1 \\
0 \\
0\end{array}$ & $\begin{array}{l}m \\
\infty \\
1 \\
1 \\
\dot{m}\end{array}$ & $\bar{q}$ \\
\hline 胥 & 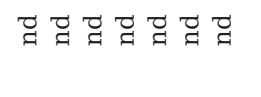 & $\Xi$ & $\widetilde{q}$ & is & 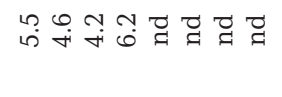 & $\bar{q}$ & $\bar{q}$ & $\bar{q}$ & $\stackrel{0}{n}$ & $\stackrel{\infty}{\infty}$ & $\stackrel{?}{\rightarrow}$ & in & $\bar{q}$ \\
\hline 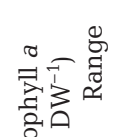 & 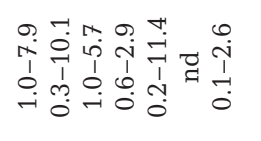 & $\begin{array}{l}-1 \\
0 \\
0 \\
0\end{array}$ & $\stackrel{2}{0}$ & $\begin{array}{l}\vec{p} \\
\dot{1} \\
\qquad \\
0 \\
\dot{0} \\
v\end{array}$ & 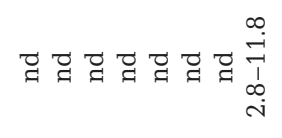 & $\stackrel{\circ}{i}$ & 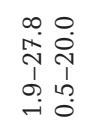 & 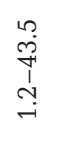 & $\begin{array}{l}0 \\
0 \\
\infty \\
1 \\
1 \\
0 \\
0\end{array}$ & $\begin{array}{l}\stackrel{1}{0} \\
i \\
1 \\
\infty \\
0 \\
0\end{array}$ & $\begin{array}{l}\infty \\
0 \\
1 \\
\stackrel{0}{0} \\
0\end{array}$ & $\begin{array}{l}\stackrel{+}{i} \\
i \\
\stackrel{i}{i}\end{array}$ & $\begin{array}{l}\not 1 \\
\stackrel{1}{1} \\
1 \\
0 \\
0\end{array}$ \\
\hline 己ี & 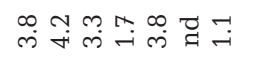 & $\stackrel{0}{0}$ & $\stackrel{\leftrightarrow}{0}$ & 苞 & 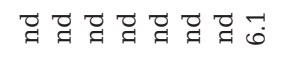 & $\stackrel{\circ}{i}$ & 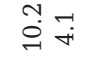 & $\stackrel{m}{\rightarrow}$ & $\stackrel{m}{=}$ & $\stackrel{\varphi}{-}$ & $\stackrel{n}{0}$ & $\stackrel{\infty}{i}$ & $\stackrel{m}{0}$ \\
\hline 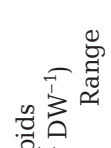 & 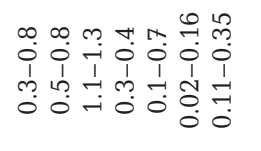 & 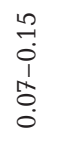 & $\vec{z}$ & $\begin{array}{l}8 \\
0 \\
0 \\
1 \\
0 \\
0 \\
0\end{array}$ & $\bar{q} \bar{q} \bar{q}$ & $\stackrel{0}{0}$ & 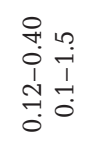 & $\begin{array}{l}\stackrel{4}{i} \\
\text { i } \\
0 \\
0\end{array}$ & $\begin{array}{l}0 \\
0 \\
1 \\
0 \\
0 \\
0\end{array}$ & $\begin{array}{l}\stackrel{n}{0} \\
0 \\
1 \\
0 \\
0\end{array}$ & $\begin{array}{l}\hat{\infty} \\
0 \\
1 \\
0 \\
0\end{array}$ & 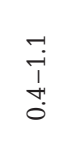 & $\begin{array}{l}\infty \\
0 \\
0 \\
1 \\
0 \\
0 \\
0\end{array}$ \\
\hline 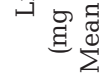 & 等菅 & 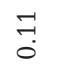 & $\overrightarrow{-1}$ & $\stackrel{n}{0}$ & 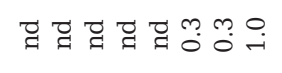 & $\stackrel{0}{0}$ & สิ ? & $\stackrel{\infty}{0}$ & $\ddot{0}$ & $\stackrel{0}{0}$ & $\stackrel{n}{0}$ & $\stackrel{\infty}{0}$ & $\overrightarrow{\tilde{o}}$ \\
\hline 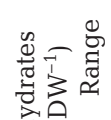 & 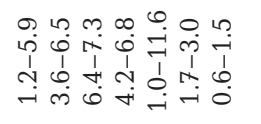 & $\begin{array}{l}0 \\
\stackrel{1}{1} \\
0 \\
0 \\
0\end{array}$ & $\stackrel{\varpi}{\nexists}$ & 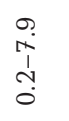 & 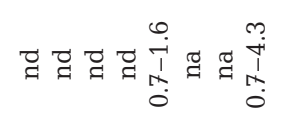 & $\stackrel{\pi}{\sharp}$ & 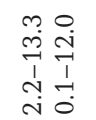 & 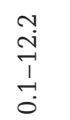 & $\begin{array}{l}\hat{a} \\
\stackrel{i}{I} \\
\overrightarrow{0}\end{array}$ & $\begin{array}{l}0 \\
\dot{1} \\
1 \\
\stackrel{9}{-} \\
-\end{array}$ & $\underset{\substack{1 \\
\rightarrow \\
I \\
0 \\
0 \\
0}}{1}$ & 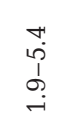 & $\begin{array}{l}\infty \\
0 \\
1 \\
1 \\
0 \\
0\end{array}$ \\
\hline 己 & 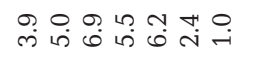 & $\stackrel{n}{0}$ & $\stackrel{\infty}{\circ}$ & $\stackrel{?}{\rightarrow}$ & 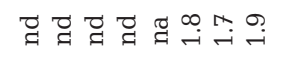 & $\stackrel{m}{0}$ & 事另 & $\stackrel{\infty}{\infty}$ & $\underset{\infty}{\infty}$ & $\stackrel{m}{+}$ & $\stackrel{\infty}{+}$ & $\stackrel{\infty}{\dot{m}}$ & : \\
\hline 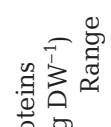 & 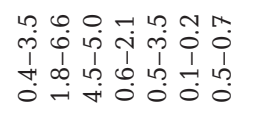 & $\begin{array}{l}m \\
0 \\
i \\
i \\
0\end{array}$ & ชั & $\begin{array}{l}\stackrel{0}{1} \\
\stackrel{1}{1} \\
\stackrel{0}{0}\end{array}$ & 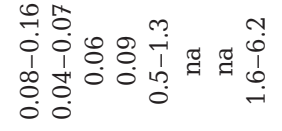 & $\stackrel{0}{i}$ & 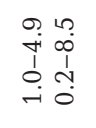 & $\begin{array}{l}0 \\
\stackrel{+}{1} \\
\stackrel{1}{0}\end{array}$ & 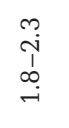 & $\begin{array}{l}0 \\
i \\
i \\
i \\
0\end{array}$ & \begin{tabular}{l}
\multirow{1}{1}{} \\
$\stackrel{1}{1}$ \\
$\stackrel{0}{0}$
\end{tabular} & 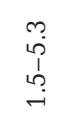 & $\begin{array}{l}\ddot{H} \\
\stackrel{p}{1} \\
\tilde{o}\end{array}$ \\
\hline$\sim$ 要吾 & 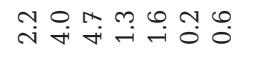 & กุ & กุ & $\stackrel{\circ}{-}$ & 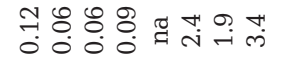 & $\stackrel{\leftrightarrow}{i}$ & $\stackrel{\infty}{\rightarrow+\infty}$ & $\stackrel{n}{\rightarrow}$ & $\stackrel{\circ}{i}$ & $\stackrel{-}{-}$ & $\stackrel{g}{0}$ & $\stackrel{\circ}{\dot{m}}$ & $\vec{i}$ \\
\hline 营 & 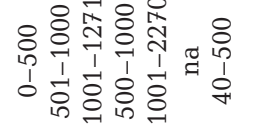 & 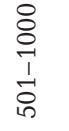 & 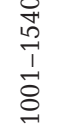 & 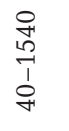 & 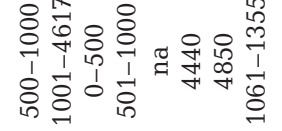 & 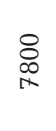 & $\begin{array}{l}\underset{N}{N} \\
1 \\
\infty \\
\infty\end{array}$ & $\begin{array}{l}0 \\
\text { in } \\
\text { ô }\end{array}$ & 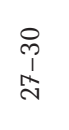 & ஓे & $\begin{array}{l}\text { â } \\
\vdots \\
\stackrel{N}{N}\end{array}$ & $\begin{array}{l}\infty \\
\tilde{n} \\
0 \\
\tilde{m}\end{array}$ & $\begin{array}{l}0 \\
0 \\
0 \\
1 \\
\stackrel{m}{+}\end{array}$ \\
\hline 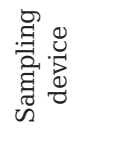 & 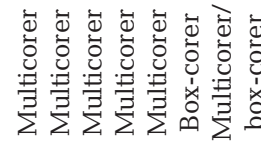 & 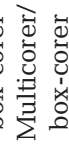 & 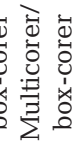 & 崩 & 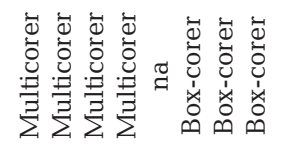 & & & & 至 & 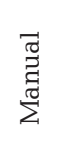 & 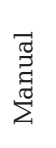 & 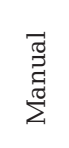 & 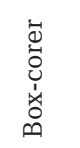 \\
\hline 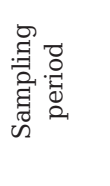 & 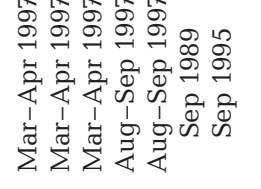 & 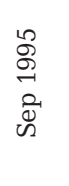 & 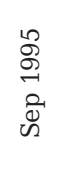 & 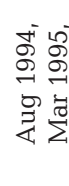 & 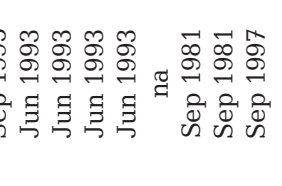 & $\begin{array}{l}\text { के } \\
\stackrel{2}{2} \\
\frac{2}{2}\end{array}$ & 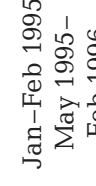 & 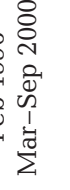 & 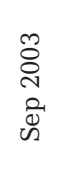 & 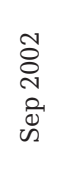 & 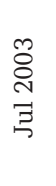 & 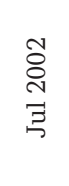 & 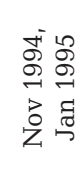 \\
\hline 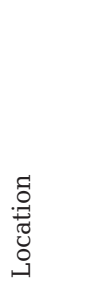 & 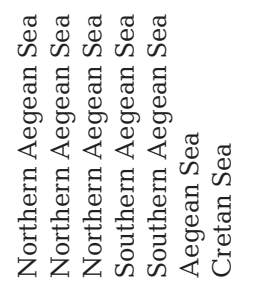 & 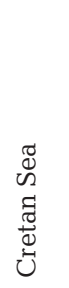 & 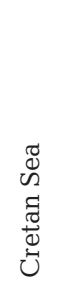 & 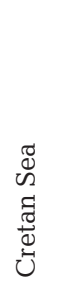 & 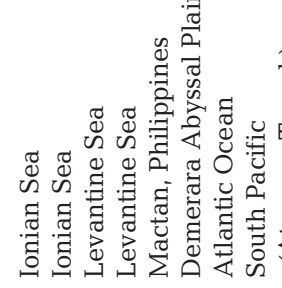 & 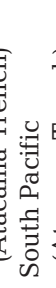 & 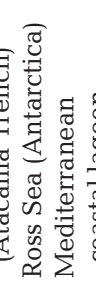 & & 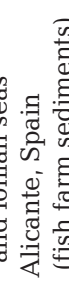 & 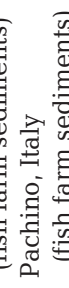 & 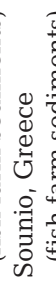 & 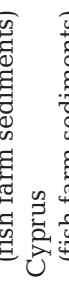 & 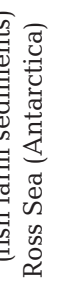 \\
\hline
\end{tabular}


Systematic variations in BPC and TOC concentrations and temporal variability in concentration of BPC in different ecosystems were assessed as the value of mean squares after a 1-way ANOVA was performed using sampling time as a single source of variance, separately for each data set.

\section{PRIMARY PRODUCTION AND SEDIMENT ORGANIC ENRICHMENT}

Relatively few studies have reported synoptic determinations of TOC and BPC concentrations. Using a reduced data set available from a few studies carried out in the Mediterranean Sea, we found that BPC is significantly related to TOC (Fig. 1). The BPC represents $71 \%$ of the TOC variance, while the remaining $29 \%$ is ascribed to other factors such as the variable composition and importance of the refractory fractions (Middelburg et al. 1999). However, whilst the biopolymeric fraction undergoes rapid changes in quantity and composition during early diagenesis, TOC generally exhibits a more conservative nature (Fabiano et al. 1995). This suggests that changes in the quantity of BPC might respond to changes and/or differences in the systems' productivity more promptly than TOC.

To test this hypothesis, we examined the relationship between proxies of primary production and BPC. The input of primary organic matter to the sea bottom (together with the benthic algal biomass, if present) is generally assessed using phytopigment concentrations in surface sediments (Pfannkuche et al. 2000). In our analysis, the total phytopigment concentrations (the sum of chl $a$ and phaeopigments, expressed as carbon equivalents) explained only about $13 \%$ of BPC variance (Type-I regression: $\mathrm{r}^{2}=0.127, \mathrm{n}=124, \mathrm{p}<0.01$ ), with the remaining $87 \%$ being ascribed to other sources, including non-marine sources, of organic matter entering the system. Since photosynthetic pigments are labile compounds (Lee et al. 2000), their concentration in surface sediments may reflect primary production processes only at shallow water depths. In contrast, their progressive depletion during particle sinking through the water column will generally result in a progressive decrease of the phytopigment concentration in deepsea sediments in moving from bathyal to abyssal depths (Fabiano et al. 2001). The weak relationship that we observed between phytopigment concentrations and BPC concentration is, therefore, potentially biased by the data collected at deeper depths. The analysis of different types of coastal ecosystems, in fact, indicates a much

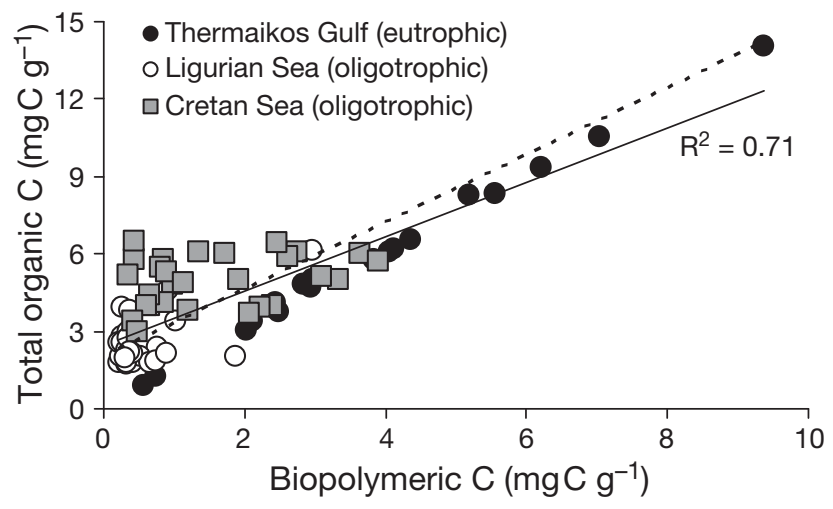

Fig. 1. Type-I (continuous line) and Type-II (dashed line) linear regressions between TOC and BPC concentrations in Mediterranean sediments. Data are extracted from Fabiano et al. (1995) for Ligurian Sea, Tselepides et al. (2000) for Cretan Sea and Pusceddu et al. (2005) for Thermaikos Gulf. $\mathrm{R}^{2}$ is the coefficient of Type-I regression

closer relationship between total phytopigment and BPC concentrations (Fig. 2a), with significant changes seen in the slope of the linear regressions (Table 2). Despite the fact that the 3 selected areas represent only part of the primary production levels found worldwide, the observed pattern suggests that the BPC in oligotrophic coastal systems (e.g. Ligurian Sea) is more closely dependent upon the input of primary organic matter than that in eutrophic systems (e.g. Adriatic Sea).

We observed decreasing algal carbon contributions to the BPC pools with increasing BPC concentrations in the sediment (Fig. 2b). The comparison of environments characterised by diverse organic matter input and different productivities indicates that increasing accumulation of BPC in marine sediments is associated even in highly productive systems (such as estuaries, ponds and fish farm sediments) with low algal carbon contributions to BPC. This suggests that, in eutrophic sediments characterised by high BPC concentrations and benthic algal biomass, algal carbon is progressively diluted in a complex and heterogeneous organic matrix. In this regard, however, it must be considered that the carbon:chl a ratio of living algae can vary from 10 to about 100 (Banse 1977). Since we used a ratio of

Table 2. Output of the analysis of covariance (ANCOVA) testing for differences between slopes and intercepts of Type-I regressions illustrated in Fig. 2a. ${ }^{* * *} \mathrm{p}<0.001,{ }^{*} \mathrm{p}<0.05, \mathrm{~ns}=$ not significant, na $=$ not applicable

\begin{tabular}{|c|c|c|c|c|}
\hline \multirow[b]{2}{*}{ ANCOVA comparison } & \multicolumn{2}{|c|}{$-F$} & \multicolumn{2}{|c|}{ Multiple comparisons } \\
\hline & Slope & Intercept & Slope & Intercept \\
\hline Liguran Sea (L) vs Ross Sea (R) & $32.20^{* * *}$ & $0.37 \mathrm{~ns}$ & $\mathrm{~L}>\mathrm{R}$ & na \\
\hline Ross Sea (R) vs Adriatic Sea (A) & $4.21^{*}$ & $5.86^{*}$ & $\mathrm{R}>\mathrm{A}$ & $\mathrm{R}<\mathrm{A}$ \\
\hline Ligurian Sea (L) vs Adriatic Sea (A) & $4.04^{*}$ & $0.006 \mathrm{~ns}$ & $\mathrm{~L}>\mathrm{A}$ & na \\
\hline
\end{tabular}



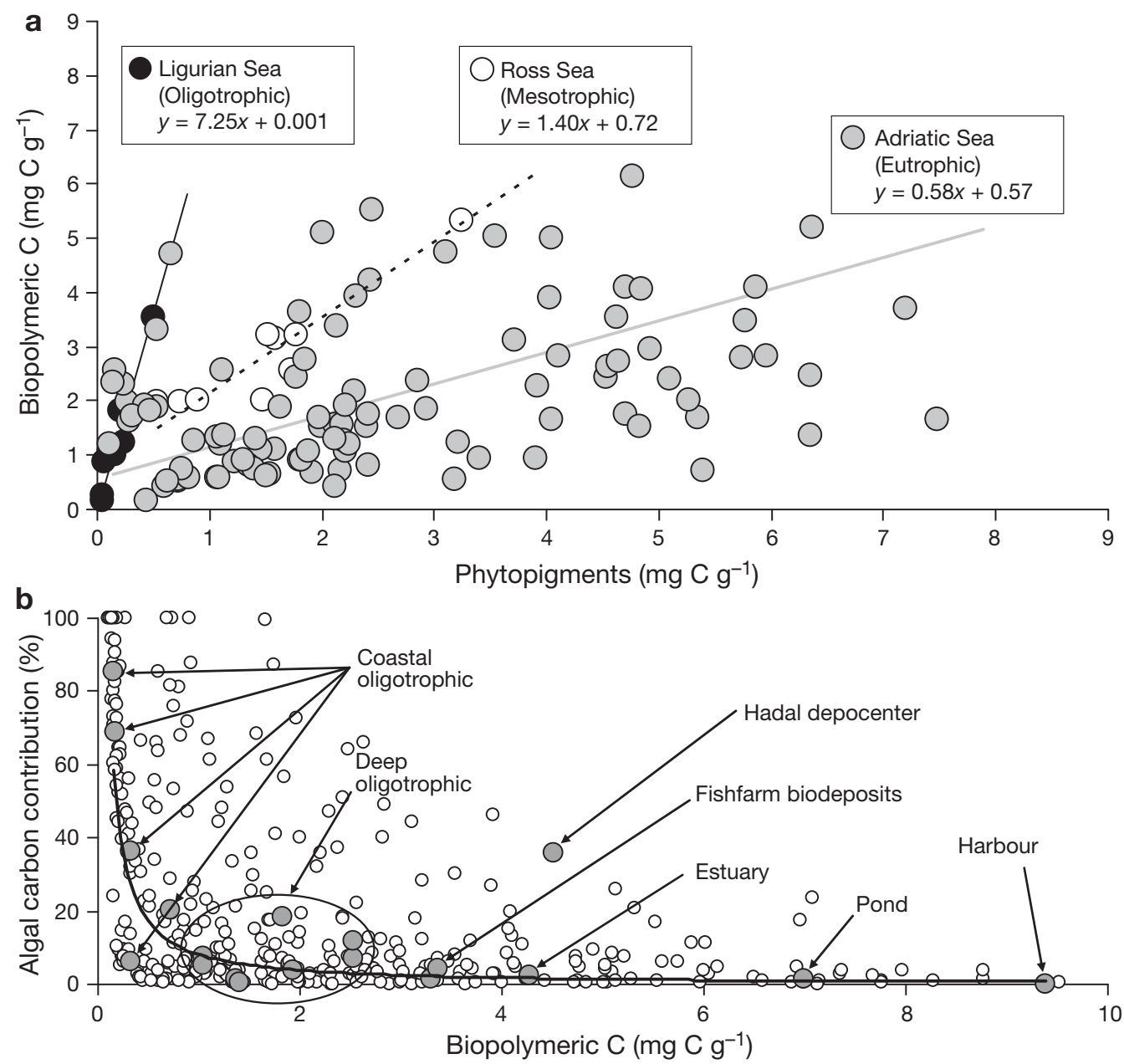

Fig. 2. Type-II regressions between phytopigment ( $\mathrm{chl} a$ and phaeopigment in C equivalents) and BPC concentrations in marine sediments in 3 coastal ecosystems with (a) different primary productivity levels, and (b) relationship between the fraction of BPC that is made up by phytopigments (= algal carbon contribution, expressed as the percentage of chl a carbon equivalents) and BPC concentrations in marine sediments. Data for (a) are extracted from Fabiano et al. (1995) for Ligurian Sea, Pusceddu et al. (2000) for Ross Sea and Danovaro et al. (2002) for Adriatic Sea. In (b), grey circles represent the mean values of representative sediments characterised by increasing BPC concentrations, whereas open circles represent all data

40 , our estimates of the algal carbon contribution to BPC are potentially biased by a factor of up to 2 to 4 . Moreover, we compared systems characterised by a wide variability in depositional regimes, so that the values of the algal carbon contribution to BPC should be considered with some caution.

Sediments exhibiting different BPC loads are also characterised by different temporal variability patterns. Marine sediments characterised by high BPC loads, such as coastal lagoons and organically enriched sediments beneath cages at fish farms, display a more pronounced temporal variability. Conversely, benthic systems characterised by lower BPC contents are characterised by a much lower temporal variability (Fig. 3). This result suggests that under 'eutrophic' conditions (i.e. high primary productivity) the inputs of organic carbon widely fluctuate, determining strong and sud- den shifts of background levels of sediment organic matter concentrations. Conversely, in oligotrophic systems, the seasonal organic carbon input during algal blooms does not detectably alter background concentrations of organic matter in the sediment.

\section{BIOPOLYMERIC CARBON AND OXYGEN DEMAND}

Organic matter reaching the sediment surface stimulates benthic metabolism (Witbaard et al. 2001). This has been used for carrying out a worldwide estimate of particulate organic carbon fluxes to the deep ocean (>1000 $\mathrm{m}$ in depth) from benthic oxygen flux estimates (Jahnke 1996). In turn, the accumulation of organic carbon in the sediment is also a function of organic carbon reactivity with the available oxygen (Dauwe et al. 

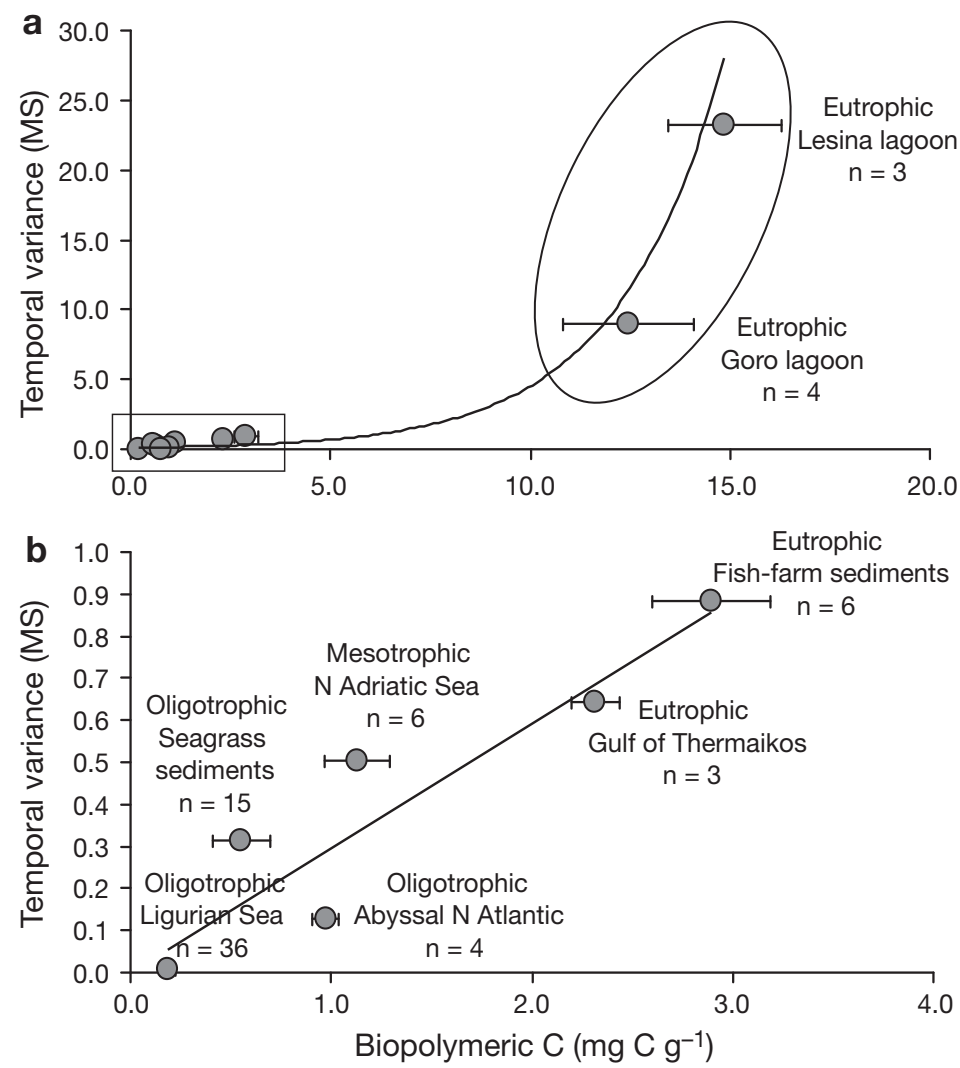

Fig. 3. Relationship between BPC sediment content and its temporal variability in different marine environments. Temporal variability is reported as mean squares (MS) derived after a 1-way ANOVA performed using sampling time as a single source of variance, separately for each data set; (b) is an enlarged view of the inset in (a). Horizontal bars indicate SE values. Data plotted refer to Manini et al. (2003) for coastal lagoons, Mazzola et al. (1999) for fish farm sediments, Pusceddu et al. (2005) for Thermaikos Gulf, Danovaro et al. (2002) for N Adriatic Sea), Danovaro et al. (1994) for seagrass sediments, Fabiano et al. (1995) for Ligurian Sea, and Danovaro et al. 2001 for Atlantic sediments

2001). When the sediment experiences an excessive organic input, oxygen can be completely depleted, with anaerobic processes prevailing.

We have found significant relationships between in situ measurements of SCOC and synoptic measurements of TOC and BPC concentration of the sediment (Fig. 4). The results of Type-I regression indicated that concentrations of both pools explained significant proportions of the variance of oxygen consumption rates, but whilst total organic carbon explained $61 \%$ of SCOC variance, BPC alone can explain $79 \%$ of SCOC variance. From the linear equations calculated using Type-II regression, it can be calculated that an increase of $1 \mathrm{~mol} \mathrm{C} \mathrm{m}{ }^{-2}$ in sediment BPC concentration would enhance SCOC by about $100 \mu \mathrm{mol} \mathrm{m} \mathrm{m}^{-2} \mathrm{~h}^{-1}$, which is about 2-fold higher than the SCOC increase obtained by an equal increase in TOC concentration (about $53 \mu \mathrm{mol} \mathrm{m} \mathrm{m}^{-2} \mathrm{~h}^{-1}$ ). These results indicate that the $\mathrm{BPC}$ is more labile towards degradation than is the total carbon pool and suggest that an enrichment in the BPC concentration, more than in TOC, will shift the system towards conditions of higher oxygen demand.

The ecological consequences of excessive organic accumulation in the sediment will also depend upon the hydrodynamic regime and other environmental constraints (e.g. water mixing, temperature, salinity, pressure, biological activity), to which the remaining 21 to $39 \%$ of the unexplained variance in the relationships outlined previously can be ascribed. Nonetheless, our results suggest that high BPC concentrations in surface sediments might be an additional co-factor potentially responsible for the development of hypoxic and/or anoxic events (Dell'Anno et al. 2008).

\section{BIOPOLYMERIC AND BIOAVAILABLE ORGANIC CARBON RELATIONSHIPS: TROPHIC CONSEQUENCES}

Since BPC may contain molecules that become available to consumers only after microbial reworking, the use of BPC as a proxy for labile organic carbon depends also on its origin and molecular composition. The amount of labile (bioavailable) organic matter in marine sediments can be extrapolated according to
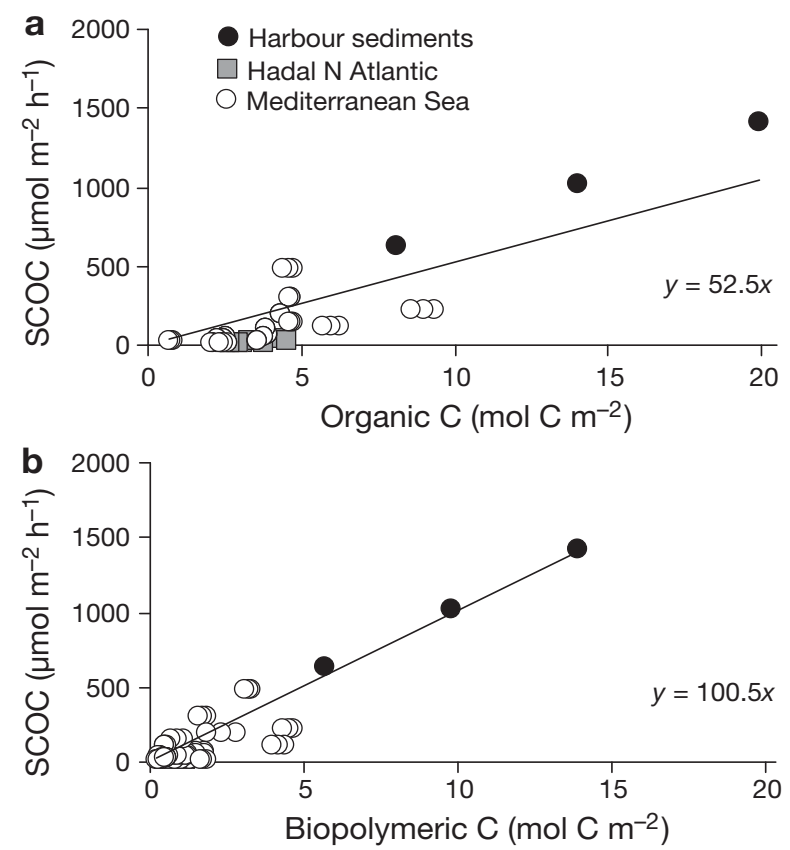

Fig. 4. Relationships between sediment community oxygen consumption (SCOC) and (a) total organic carbon and (b) BPC concentrations in the sediment. Data are summarised from Danovaro et al. (1999a, 2002), Witbaard et al. (2000), Duineveld et al. (2000), Tselepides et al. (2000) and Lykousis et al. (2002). Type-II regression equations are reported 
mathematical models based on the exponential decrease of organic carbon with increasing depth in the sediments (Rice \& Rhoads 1989). Such models, however, assume that different classes of organic compounds undergo degradation and utilisation at similar rates (Danovaro et al. 2001).

A number of studies demonstrated that only a certain fraction of BPC in marine sediments can actually be degraded enzymatically and, thus, is readily available for heterotrophic nutrition (Dauwe \& Middelburg 1998, Dauwe et al. 1999a,b, Dell'Anno et al. 2000, Danovaro et al. 2001). Based on this background, we investigated the relationship between concentrations of BPC and bioavailable carbon (BAC) in sediments characterised by different productivity levels (Fig. 5a). As expected from the linear relationship between BPC and the sediment community oxygen consumption, BAC is positively correlated with BPC, suggesting that eutro- phic (i.e. enriched in BPC concentration) sediments are characterised also by high concentrations of rapidly digestible material. However, increasing BPC concentrations in the sediment also display a progressive decrease of the percentage contribution of the bioavailable carbon to BPC (Fig. 5b). This finding would indicate that benthic environments subjected to changes in trophic status may experience relevant changes in the relative importance of the labile and refractory fractions of sediment organic carbon. These changes could be also the result of differences in sediment particle characteristics, which are known to play a major role in the preservation (and, thus, lability) of the different pools of sediment organic matter (Keil et al. 1994). Nevertheless, our results are in agreement with previous studies indicating that eutrophic systems, subjected to the accumulation of large amounts of organic matter in subsurface sediments, could be
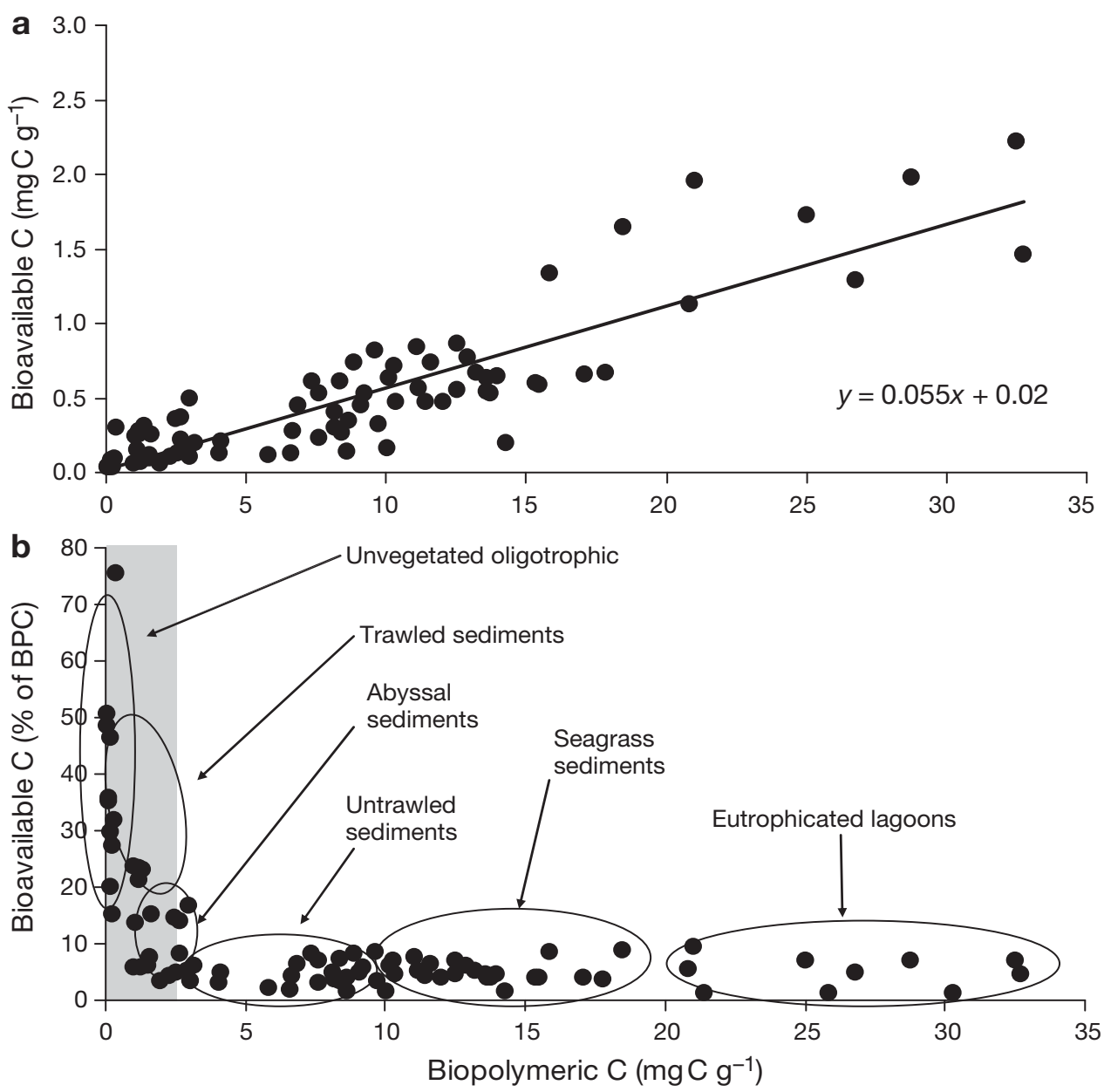

Fig. 5. Relationships between (a) BPC and BAC quantities and (b) the bioavailable (enzymatically digestible) fraction of BPC versus BPC quantity in marine sediments. Data are extracted from Danovaro et al. (2001) for the Porcupine Abyssal Plain (4850 m depth), Pusceddu et al. (2003) for the western Mediterranean Sea (2 m depth), Pusceddu et al. (2005) for the eastern Mediterranean Sea (30 to $86 \mathrm{~m}$ depth) and Pusceddu et al. (2007b) for the Goro and Lesina lagoons. The light grey area encloses values of $\mathrm{BPC}$ concentrations less than $2.5 \mathrm{mg} \mathrm{C} \mathrm{g}^{-1}$, which correspond to values of the bioavailable fraction $>10 \%$. The equation in (a) is derived by Type-II regression analysis 
characterised by an enhancement of the complexation of buried organic molecules with the inorganic matrix, thus making them less 'available' to heterotrophic nutrition (the so-called 'sorptive preservation hypothesis'; Mayer 1994). This could be also due to an 'encapsulation' mechanism of potentially labile molecules (such as proteins) in organically enriched sediments (such as those buried in the sapropel layers; Knicker \& Hatcher 1997). The accumulation of mostly refractory organic matter in organically enriched sediments appears to be the case of transitional (lagoonal) ecosystems (e.g. Pusceddu et al. 2003).

From these relationships, it can also be seen that when BPC concentrations in the sediment exceed $2.5 \mathrm{mg} \mathrm{C} \mathrm{g}^{-1}$, its bioavailable fraction is always less than $10 \%$. These 2 values, when verified contemporarily in the same area, can, thus, be proposed as threshold levels out of which accumulation of BPC leads to altered organic matter bioavailability to benthic consumers.

Acknowledgements. This study was undertaken in the framework of the PR1 project funded by the Italian Ministry of the Environment, and with NITIDA (COFIN2003) funding provided by the Italian Ministry of University and Research. We are grateful to G. Sarà, S. Fraschetti and M. A. Colangelo for help in statistical analyses and 4 anonymous reviewers who gave constructive comments on early versions of the manuscript.

\section{LITERATURE CITED}

Albertelli G, Covazzi-Harriague A, Danovaro R, Fabiano M, Fraschetti S, Pusceddu A (1999) Differential responses of bacteria, meiofauna and macrofauna in a shelf area (Ligurian Sea, NW Mediterranean): role of food availability. J Sea Res 42:11-26

Banse K (1977) Determining the carbon-to-chlorophyll ratio of natural phytoplankton. Mar Biol 41:199-212

Berges JA, Fisher AE, Harrison PJ (1993) A comparison of Lowry, Bradford and Smith protein assays using different protein standards and protein isolated from the marine diatom Thalassiosira pseudonana. Mar Biol 115:187-193

Bianchelli S, Gambi C, Pusceddu A, Danovaro R (2008) Trophic conditions and meiofaunal assemblages in the Bari Canyon and the adjacent open slope (Adriatic Sea). Chem Ecol 24(S1):101-109

Bligh EG, Dyer WJ (1959) A rapid method for total lipid extraction and purification. Can J Biochem Physiol 37: 911-917

Boetius A, Scheibe S, Tselepides A, Thiel H (1996) Microbial biomass and activities in deep-sea sediments of the Eastern Mediterranean: trenches as benthic hotspots. Deep-Sea Res I 104:299-307

Bradford MM (1976) A rapid and sensitive method for the quantitation of microgram quantities of protein utilizing the principle of protein-dye binding. Anal Biochem 72: 248-254

Buscail R, Pocklington R, Germain C (1995) Seasonal variability of the organic matter in a sedimentary coastal environment: sources, degradation and accumulation (continental shelf of the Gulf of Lions-northwestern Mediterranean Sea). Cont Shelf Res 15:843-869

Cebrián J, Williams M, McClelland J, Valiela I (1998) The dependence of heterotrophic consumption and $\mathrm{C}$ accumulation on autotrophic nutrient concentration in ecosystems. Ecol Lett 1:165-170

Cividanes S, Incera M, Lopez J (2002) Temporal variability in the biochemical composition of sedimentary organic matter in an intertidal flat of the Galician coast (NW Spain). Oceanol Acta 25:1-12

> Cloern JE (2001) Our evolving conceptual model of the coastal eutrophication problem. Mar Ecol Prog Ser 210: $223-253$

> Colombo JC, Silverberg N, Gearing JN (1996) Biogeochemistry of organic matter in the Laurentian Trough, 2. Bulk composition of the sediments and relative reactivity of major components during early diagenesis. Mar Chem 51: 295-314

Cornwell JC, Conley DJ, Owens M, Stevenson JC (1996) A sediment chronology of the eutrophication of Chesapeake Bay. Estuaries 19:488-499

> Danovaro R, Fabiano M (1997) Seasonal changes in quality and quantity of food available for benthic suspensionfeeders in the Golfo Marconi (North-western Mediterranean). Estuar Coast Shelf Sci 44:723-736

Danovaro R, Fabiano M, Della Croce N (1993) Labile organic matter and microbial biomasses in deep-sea sediments (Eastern Mediterranean Sea). Deep-Sea Res I 40:953-965

> Danovaro R, Fabiano M, Boyer M (1994) Seasonal changes of benthic bacteria in a seagrass (Posidonia oceanica) bed in relation to the origin, composition and fate of the sediment organic matter. Mar Biol 119:489-500

Danovaro R, Della Croce N, Eleftheriou A, Fabiano M, Papadopoulou N, Smith C, Tselepides A (1995) Meiofauna of the deep Eastern Mediterranean Sea: distribution and abundance in relation to bacterial biomass, organic matter composition and other environmental factors. Prog Oceanogr 36:329-341

> Danovaro R, Marrale D, Della Croce N, Dell'Anno A, Fabiano M (1998) Heterotrophic nanoflagellates, bacteria, and labile organic compounds in continental shelf and deep-sea sediments of the eastern Mediterranean. Microb Ecol 35: 244-255

Danovaro R, Dinet A, Duineveld G, Tselepides A (1999a) Benthic response to particulate fluxes in different trophic environments: a comparison between the Gulf of LionsCatalan Sea (western-Mediterranean) and the Cretan Sea (eastern-Mediterranean). Prog Oceanogr 44:287-312

> Danovaro R, Marrale D, Della Croce N, Parodi P, Fabiano M (1999b) Biochemical composition of sedimentary organic matter and bacterial distribution in the Aegean Sea: trophic state and pelagic-benthic coupling. J Sea Res 42: $117-129$

Danovaro R, Pusceddu A, Covazzi Harriague A, Marrale D and others (1999c) Community experiments using benthic chambers: microbial significance in highly organic enriched sediments. Chem Ecol 16:7-30

Danovaro R, Dell'Anno A, Fabiano M (2001) Bioavailability of organic matter in the sediments of the Porcupine Abyssal Plain. Mar Ecol Prog Ser 220:25-32

Danovaro R, Manini E, Fabiano M (2002) Exoenzymatic activity and organic matter composition in sediments of the Northern Adriatic Sea: response to a river plume. Microb Ecol 44:235-251

Danovaro R, Della Croce N, Dell'Anno A, Pusceddu A (2003) A depocenter of organic matter at $7800-\mathrm{m}$ depth in the SE Pacific Ocean. Deep-Sea Res I 50:1411-1420 
Dauwe B, Middelburg JJ (1998) Amino acids and hexosamines as indicators of organic matter degradation state in North Sea sediments. Limnol Oceanogr 43:782-798

> Dauwe B, Middelburg JJ, Van-Rijswijk P, Sinke J, Herman PMJ, Heip CHR (1999a) Enzymatically hydrolyzable amino acids in North Sea sediments and their possible implication for sediment nutritional values. J Mar Res 57: 109-134

Dauwe B, Middelburg JJ, Herman PMJ, Heip CHR (1999b) Linking diagenetic alteration of amino acids and bulk organic matter reactivity. Limnol Oceanogr 44:1809-1814

$>$ Dauwe B, Middelburg JJ, Herman PMJ (2001) Effect of oxygen on the degradability of organic matter in subtidal and intertidal sediments of the North Sea area. Mar Ecol Prog Ser 215:13-22

> Dell'Anno A, Fabiano M, Mei ML, Danovaro R (2000) Enzymatically hydrolysed protein and carbohydrates pools in deep-sea sediments: estimates of the bioavailable fraction and methodological considerations. Mar Ecol Prog Ser 196: $15-23$

> Dell'Anno A, Mei ML, Pusceddu A, Danovaro R (2002) Assessing the trophic state and eutrophication of coastal marine systems: a new approach based on the biochemical composition of sediment organic matter. Mar Pollut Bull 44:611-622

Dell'Anno A, Pusceddu A, Langone L, Danovaro R (2008) Biochemical composition and early diagenesis of organic matter in coastal sediments of the NW Adriatic Sea influenced by riverine inputs. Chem Ecol 24:75-85

Duarte CM (1995) Submerged aquatic vegetation in relation to different nutrient regimes. Ophelia 41:87-112

> Duijnstee I, de Lugt I, Vonk Noordegraaf H, van der Zwaan B (2004) Temporal variability of foraminiferal densities in the northern Adriatic Sea. Mar Micropaleontol 50:125-148

> Duineveld GCA, Tselepides A, Witbaard R, Bak RPM and others (2000) Benthic-pelagic coupling in the oligotrophic Cretan Sea. Prog Oceanogr 46:457-481

Emeis KC, Struck U, Leipe T, Pollehne F, Kunzendorf H, Christiansen C (2000) Changes in the C, N, P burial rates in some Baltic Sea sediments over the last 150 years - relevance to $\mathrm{P}$ regeneration rates and the phosphorus cycle. Mar Geol 167:43-59

Fabiano M, Danovaro R (1994) Composition of organic matter in sediments facing a river estuary (Tyrrhenian Sea): relationships with bacteria and microphytobenthic biomass. Hydrobiologia 277:71-84

Fabiano M, Danovaro R (1998) Enzymatic activity bacterial distribution and organic matter composition in sediments of the Ross Sea (Antarctica). Appl Environ Microbiol 64: 3838-3845

Fabiano M, Danovaro R, Fraschetti S (1995) A three-year time series of elemental and biochemical composition of organic matter in subtidal sandy sediments of the Ligurian Sea (northwestern Mediterranean). Cont Shelf Res 15: 1453-1469

Fabiano M, Pusceddu A, Dell'Anno A, Armeni M and others (2001) Fluxes of phytopigments and labile organic matter to the deep ocean in the NE Atlantic Ocean. Prog Oceanogr 50:89-104

Farías L (2003) Remineralization and accumulation of organic carbon and nitrogen in marine sediments of eutrophic bays: the case of the Bay of Concepcion, Chile. Estuar Coast Shelf Sci 57:829-841

Fichez R (1991) Composition and fate of organic matter in submarine cave sediments: implications for the biogeochemical cycle of organic carbon. Oceanol Acta 14:369-377

Gerchacov SM, Hatcher PG (1972) Improved technique for analysis of carbohydrates in sediment. Limnol Oceanogr 17:938-943

Graf G, Meyer Reil LA (1985) Remineralization of organic substances on benthic surfaces in the intertidal reef area off Mactan, Cebu, Philippines. Philippine Sci 22:42-46

Grall J, Chauvaud L (2002) Marine eutrophication and benthos: the need for new approaches and concepts. Glob Change Biol 8:813-830

Hartree EF (1972) Determination of proteins: a modification of the Lowry method that gives a linear photometric response. Anal Biochem 48:422-427

Hedges JI, Stern JH (1984) Carbon and nitrogen determination of carbonate-containing solids. Limnol Oceanogr 29: $657-663$

- Huxel GR (1999) On the influence of food quality in consumer-resource interactions. Ecol Lett 2:256-261

Jahnke RA (1996) The global ocean flux of particulate organic carbon: a real distribution and magnitude. Glob Biogeochem Cycles 10:71-88

> Jolicoeur P (1990) Bivariate allometry: interval estimation of the slopes of the ordinary and standardized normal major axes and structural relationship. J Theor Biol 144:275-285

Jørgensen BB, Richardson K (1996) Eutrophication in coastal marine ecosystems. Coastal and Estuarine Studies 52, Am Geophys Union, Washington, DC

Keil RG, Montluçon DB, Prahl FG, Hedges JI (1994) Sorptive preservation of labile organic matter in marine sediments. Nature 370:549-552

Kelly MG (1998) Use of the yrophic diatom index to monitor eutrophication in rivers. Water Res 32:236-242

Khripounoff A, Crassous P, Desbruyeres D, Le Cox JR (1985) Le flux organique particulaire et ses transformations a l'interface eau-sediment. In: Laubier L, Monniot C (eds) Peuplements profonds du Golfe de Gascogne. IFREMER, France, p 101-118

- Knicker H, Hatcher P (1997) Survival of protein in an organicrich sediment: possible protection by encapsulation in organic matter. Naturwissenschaften 84:231-234

Lee C, Wakeham SG, Hedges JI (1988) The measurement of oceanic particulate flux: Are swimmers a problem? Oceanography 2:34-36

Lee C, Wakeham SG, Hedges JI (2000) Composition and flux of particulate amino acids and chloropigments in equatorial Pacific seawater and sediments. Deep-Sea Res I 47:1535-1568

Legendre P (2001) Model II regression-user's guide. Département de sciences biologiques, Université de Montréal

Legendre P, Legendre L (1998) Numerical ecology, 2nd English edn. Elsevier Science BV, Amsterdam

Lorenzen CJ, Jeffrey SW (1980) Determination of chlorophyll and phaeopigments spectrophotometric equations. Limnol Oceanogr 12:343-346

Lowry OH, Rosenbrough NS, Farr AL, Randall RJ (1951) Protein measurement with the Folin-phenol reagent. J Biol Chem 193:265-275

Lykousis V, Chronis G, Tselepides A, Price NB and others (2002) Major outputs of the recent multidisciplinary biogeochemical researches undertaken in the Aegean Sea. J Mar Syst 33-34:313-334

Manini E, Fiordelmondo C, Gambi C, Pusceddu A, Danovaro $\mathrm{R}$ (2003) Benthic microbial loop functioning in coastal lagoons: a comparative approach. Oceanol Acta 26:27-38

> Marsh JB, Weinstein DB (1966) A simple charring method for determination of lipids. J Lipid Res 7:574-576

Mayer LM (1994) Surface area control of organic carbon accumulation in continental shelf sediments. Geochim Cosmochim Acta 58:1271-1284 
Mayer LM, Schick LL, Sawyer T, Plante CJ, Jumars PA, Self RL (1995) Bioavailable amino acids in sediments: a biomimetic, kinetics-based approach. Limnol Oceanogr 40: 511-520

Mazzola A, Mirto S, Danovaro R (1999) Initial fish-farm impact on meiofaunal assemblages in coastal sediments of the Western Mediterranean. Mar Pollut Bull 38:1126-1133

Meyer Reil LA (1983) Benthic response to sedimentation events during autumn to spring at a shallow water station in the Western Kiel Bight. II. Analysis of benthic bacterial populations. Mar Biol 77:247-256

Meyer Reil LA, Graf G (1986) Seasonal development of bacterial communities in a coastal marine sediment as related to the input of organic material. GERBAM - Deuxieme Colloque International de Bacteriologie Marine - CNRS, Brest, IFREMER. Actes du Colloque 3:55-59

Middelburg JJ, Nieuwenhuize J, van-Breugel P (1999) Black carbon in marine sediments. Mar Chem 65:245-252

Nixon SW (1995) Coastal marine eutrophication: a definition, social causes, and future concerns. Ophelia 41:199-219

Pfannkuche O, Thiel H (1987) Meiobenthic stocks and benthic activity on the NE-Svalbard shelf and in the Nansen Basin. Polar Biol 7:253-266

Pfannkuche O, Sommer S, Kähler A (2000) Coupling between phytodetritus depositing and the small-sized benthic biota in the deep Arabian Sea: analyses of biogenic sediment compounds. Deep-Sea Res II 47:2805-2833

Pinckney JL, Paerl HW, Tester P, Richardson TL (2001) The role of nutrient loading and eutrophication in estuarine ecology. Environ Health Perspect 109:699-706

Pusceddu A, Sarà G, Armeni M, Fabiano M, Mazzola A (1999) Seasonal and spatial changes in the sediment organic matter of a semi-enclosed marine system (W-Mediterranean Sea). Hydrobiologia 397:59-70

Pusceddu A, Dell'Anno A, Fabiano M (2000) Organic matter composition in coastal sediments at Terra Nova Bay (Ross Sea) during summer 1995. Polar Biol 23:288-293

Pusceddu A, Dell'Anno A, Manini E, Fabiano M, Sarà G, Danovaro R (2003) Enzymatically hydrolyzable protein and carbohydrate sedimentary pools as indicators of the trophic state of 'detritus sink' systems: a case study in a Mediterranean coastal lagoon. Estuaries 26:641-650

Pusceddu A, Fiordelmondo C, Polymenakou P, Polychronaki T, Tselepides A, Danovaro R (2005) Effects of bottom trawling on the quantity and biochemical composition of organic matter in coastal marine sediments (Thermaikos Gulf, northwestern Aegean Sea). Cont Shelf Res 25: 2491-2505

Pusceddu A, Fraschetti S, Mirto S, Holmer M, Danovaro R (2007a) Effects of intensive mariculture on sediment biochemistry. Ecol Appl 17:1366-1378

Editorial responsibility: Matthias Seaman,

Oldendorf/Luhe, Germany
Pusceddu A, Gambi C, Manini E, Danovaro R (2007b) Trophic state, ecosystem efficiency and biodiversity of transitional aquatic ecosystems: analysis of environmental quality based on different benthic indicators. Chem Ecol 23: 505-515

Rice DL (1982) The detritus nitrogen problem: new observations and perspectives from organic geochemistry. Mar Ecol Prog Ser 9:153-162

Rice DL, Rhoads DC (1989) Early diagenesis of organic matter and the nutritional value of sediment. In: Lopez G, Taghon G, Levinton J (eds) Ecology of marine deposit-feeders. Lecture notes on coastal and estuarine studies. SpringerVerlag, New York, p 60-97

> Sampou P, Oviatt CA (1991) Seasonal patterns of sedimentary carbon and anaerobic respiration along a simulated eutrophication gradient. Mar Ecol Prog Ser 72:271-282

Shirayama Y, Fukushima T (1995) Comparisons of deep-sea sediments and overlying water collected using multiple corer and box corer. J Oceanogr 51:75-82

Sibuet M (1984) Deposit-feeding invertebrates in the deepsea ecosystem. Selectivity in feeding and diet of holothurians. Océanis 10:623-639

> Stefanou P, Tsirtsis G, Karydis M (2000) Nutrient scaling for assessing eutrophication: the development of a simulated normal distribution. Ecol Appl 10:303-309

Tselepides A, Polychronaki T, Marrale D, Akoumianaki I, Dell'Anno A, Pusceddu A, Danovaro R (2000) Organic matter composition of the continental shelf and bathyal sediments of the Cretan Sea (NE Mediterranean). Prog Oceanogr 46:311-344

Turner RE, Rabalais NN, Fry B, Atilla N and others (2006) Paleo-indicators and water quality change in the Charlotte Harbor Estuary. Limnol Oceanogr 51:518-533

Volkman JK (2006) Lipid markers for marine organic matter. In: Volkman JK (ed) Marine organic matter: biomarkers, isotopes and DNA. Springer-Verlag, Berlin, p 27-70

Witbaard R, Duineveld GCA, Van der Weele JA, Berghuis EM, Reyss JP (2000) The benthic response to the seasonal deposition of phytopigments at the Porcupine Abyssal Plain in the North East Atlantic. Prog Oceanogr 43:15-31

Witbaard R, Duineveld GCA, Kok A, van der Weele J, Berghuis EM (2001) The response of Oneirophanta mutabilis (Holothuroidea) to the seasonal deposition of phytopigments at the Porcupine Abyssal Plain in the Northeast Atlantic. Prog Oceanogr 50:423-441

Zar JH (1984) Biostatistical analysis. Prentice-Hall, Englewood Cliffs, NJ

Zimmerman AR, Canuel EA (2002) Sediment geochemical records of eutrophication in the mesohaline Chesapeake Bay. Limnol Oceanogr 47:1084-1093

Submitted: June 20, 2007; Accepted: September 12, 2008

Proofs received from author(s): January 15, 2009 